\title{
EXISTENCE RESULTS FOR CLASSES OF $p$-LAPLACIAN SEMIPOSITONE EQUATIONS
}

\author{
SHOBHA ORUGANTI AND R. SHIVAJI
}

Received 22 September 2005; Accepted 10 November 2005

We study positive $C^{1}(\bar{\Omega})$ solutions to classes of boundary value problems of the form $-\Delta_{p} u=g(x, u, c)$ in $\Omega, u=0$ on $\partial \Omega$, where $\Delta_{p}$ denotes the $p$-Laplacian operator defined by $\Delta_{p} z:=\operatorname{div}\left(|\nabla z|^{p-2} \nabla z\right) ; p>1, c>0$ is a parameter, $\Omega$ is a bounded domain in $R^{N}$; $N \geq 2$ with $\partial \Omega$ of class $C^{2}$ and connected (if $N=1$, we assume that $\Omega$ is a bounded open interval), and $g(x, 0, c)<0$ for some $x \in \Omega$ (semipositone problems). In particular, we first study the case when $g(x, u, c)=\lambda f(u)-c$ where $\lambda>0$ is a parameter and $f$ is a $C^{1}([0, \infty))$ function such that $f(0)=0, f(u)>0$ for $0<u<r$ and $f(u) \leq 0$ for $u \geq r$. We establish positive constants $c_{0}(\Omega, r)$ and $\lambda^{*}(\Omega, r, c)$ such that the above equation has a positive solution when $c \leq c_{0}$ and $\lambda \geq \lambda^{*}$. Next we study the case when $g(x, u, c)=a(x) u^{p-1}-$ $u^{\gamma-1}-\operatorname{ch}(x)$ (logistic equation with constant yield harvesting) where $\gamma>p$ and $a$ is a $C^{1}(\bar{\Omega})$ function that is allowed to be negative near the boundary of $\Omega$. Here $h$ is a $C^{1}(\bar{\Omega})$ function satisfying $h(x) \geq 0$ for $x \in \Omega, h(x) \neq 0$, and $\max _{x \in \bar{\Omega}} h(x)=1$. We establish a positive constant $c_{1}(\Omega, a)$ such that the above equation has a positive solution when $c<c_{1}$. Our proofs are based on subsuper solution techniques.

Copyright (C) 2006 S. Oruganti and R. Shivaji. This is an open access article distributed under the Creative Commons Attribution License, which permits unrestricted use, distribution, and reproduction in any medium, provided the original work is properly cited.

\section{Introduction}

We consider weak solutions to classes of boundary value problems of the form

$$
\begin{gathered}
-\Delta_{p} u=g(x, u, c) \quad \text { in } \Omega, \\
u=0 \quad \text { on } \partial \Omega,
\end{gathered}
$$

where $\Delta_{p}$ denotes the $p$-Laplacian operator defined by $\Delta_{p} z:=\operatorname{div}\left(|\nabla z|^{p-2} \nabla z\right) ; p>1, c>$ 0 is a parameter, $\Omega$ is a bounded domain in $R^{N} ; N \geq 2$ with $\partial \Omega$ of class $C^{2}$ and connected (if $N=1$, we assume that $\Omega$ is a bounded open interval) and $g(x, 0, c)<0$ for some $x \in \Omega$ (semipositone problems). By a weak solution to (1.1), we mean a function $u \in W_{0}^{1, p}(\Omega)$ 
that satisfies

$$
\int_{\Omega}|\nabla u|^{p-2} \nabla u \cdot \nabla w d x=\int_{\Omega} g(x, u, c) w d x, \quad \forall w \in C_{0}^{\infty}(\Omega) .
$$

However in this paper, we in fact study the existence of $C^{1}(\bar{\Omega})$ solutions that are strictly positive in $\Omega$.

We first study the case when $g(x, u, c)=\lambda f(u)-c$ where $\lambda>0$ is a parameter and $f$ satisfies:

(A1) $f \in C^{1}([0, \infty)), f(0)=0, f(u)>0$ for $0<u<r$ and $f(u) \leq 0$ for $u \geq r$ for some $r>0$.

When $c=0$ it is easy to establish the existence of a positive solution for large $\lambda>0$. Here we consider the challenging semipositone case $c>0$. Semipositone problems have been of great interest during the past two decades, and continue to pose mathematically difficult problems in the study of positive solutions (see [1-3, 10-12]). Also most of the results established to date are for the case when $p=2$. Here we establish an existence result for $p>1$ for a class of nonlinearities satisfying (A1). Namely, we prove the following theorem.

Theorem 1.1. There exist positive constants $c_{0}=c_{0}(\Omega, r)$ and $\lambda^{*}=\lambda^{*}(\Omega, r, c)$ such that (1.1) has a positive solution for $c \leq c_{0}$ and $\lambda \geq \lambda^{*}$.

Remark 1.2. Refer to [2] where the authors study such a problem in the case when $p=2$. In particular, when $c$ is very small they establish an existence of a positive solution for $\tilde{\lambda}$ near the first eigenvalue $\lambda_{1}$ and then extend the existence for $\lambda \geq \tilde{\lambda}$. In this paper, we establish the existence of a positive solution directly for $\lambda$ large. Our proof is new even in the case $p=2$.

Remark 1.3. The case when $g(x, u, c)=\lambda[f(u)-c]$ with $h(u)=f(u)-c$ of the form

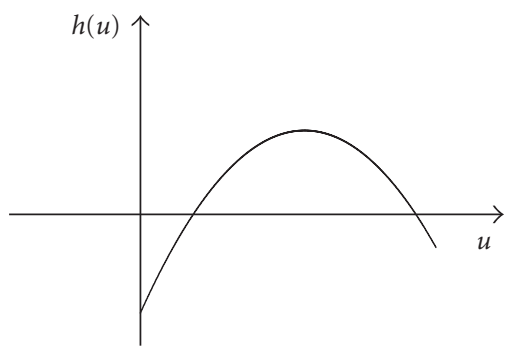

has been studied for the case when $p=2$ in [6]. For $p \neq 2$ this remains a challenging semipositone problem for existence of positive solutions for large $\lambda$.

We next study the case when $g(x, u, c)=a(x) u^{p-1}-u^{\gamma-1}-\operatorname{ch}(x)$ (Logistic equation with constant yield harvesting) where $\gamma>p, a$ is a $C^{1}(\bar{\Omega})$ function that is allowed to be negative near the boundary of $\Omega$, and $h$ is a $C^{1}(\bar{\Omega})$ function satisfying $h(x) \geq 0$ for $x \in \Omega$, $h(x) \not \equiv 0$ and $\max _{x \in \bar{\Omega}} h(x)=1$. Again for $c>0$ this is a semipositone problem. In order to precisely state our result for this problem we introduce the region where we allow $a(x)$ to be negative. Let $\lambda_{1}$ be the first eigenvalue of the $-\Delta_{p}$ with Dirichlet boundary conditions 
and $\phi_{1} \in C^{1}(\bar{\Omega})$ be a corresponding eigenfunction such that $\phi_{1}>0$ in $\Omega, \partial \phi / \partial n<0$ on $\partial \Omega$ and $\left\|\phi_{1}\right\|_{\infty}=1$. Let $m>0, \delta>0$, and $\sigma>0$ be such that

$$
\begin{gathered}
\left|\nabla \phi_{1}\right|^{p}-\lambda_{1} \phi_{1}^{p} \geq m \quad \text { on } \bar{\Omega}_{\delta}, \\
\phi_{1} \geq \sigma \quad \text { on } \Omega \backslash \bar{\Omega}_{\delta},
\end{gathered}
$$

where $\bar{\Omega}_{\delta}:=\{x \in \Omega \mid d(x, \partial \Omega) \leq \delta\}$. Further assume that there exists a constant $a_{0}>0$ such that

$$
a(x) \geq a_{0} \quad \text { in } \Omega \backslash \bar{\Omega}_{\delta}
$$

and let $\mu>0$ be such that

$$
a(x) \geq-\mu \quad \text { in } \bar{\Omega}_{\delta} .
$$

Then we prove the following theorem.

Theorem 1.4. Let $\mu<m(p /(p-1))^{p-1}$ and $a_{0}>(p /(p-1))^{p-1} \lambda_{1}$. Then there exists $a$ positive constant $c_{1}=c_{1}\left(\Omega, \mu, a_{0}\right)$ such that (1.1) has a positive solution for $c \leq c_{1}$.

Remark 1.5. Refer to [7] where they studied the case when $c=0$ and $a(x)$ is a positive function throughout $\bar{\Omega}$.

We establish Theorems 1.1 and 1.4 by the method of sub- and super-solutions. By a super-solution $\phi$ of $(1.1)$ we mean a function in $W^{1, p}(\Omega) \cap C(\bar{\Omega})$ such that $\phi=0$ on $\partial \Omega$ and

$$
\int_{\Omega}|\nabla \phi|^{p-2} \nabla \phi \cdot \nabla w d x \geq \int_{\Omega} g(x, \phi, c) w d x, \quad \forall w \in W
$$

where $W=\left\{v \in C_{0}^{\infty}(\Omega) \mid v \geq 0\right.$ in $\left.\Omega\right\}$. And by a subsolution $\psi$ of (1.1) we mean a function in $W^{1, p}(\Omega) \cap C(\bar{\Omega})$ such that $\psi=0$ on $\partial \Omega$ and

$$
\int_{\Omega}|\nabla \psi|^{p-2} \nabla \psi \cdot \nabla w d x \leq \int_{\Omega} g(x, \psi, c) w d x, \quad \forall w \in W
$$

where $W$ is as defined before. Then if there exist sub- and super-solutions $\psi$ and $\phi$ respectively such that $\psi \leq \phi$ in $\Omega$ then $(1.1)$ has a $C^{1}(\bar{\Omega})$ solution $u$ such that $\psi \leq u \leq \phi$ (see $[7,8])$.

In semipositone problems it is well documented that finding a nonnegative subsolution is nontrivial. Recently in [4] an anti-maximum principle by $[5,8,9]$ was used to create a crucial subsolution in the study of the problem when $g(x, u, c)=\lambda \tilde{f}(u)-c$ where $\tilde{f}$ satisfies $\tilde{f}(0)=0, \tilde{f}(u) \geq 0$ and $\lim _{u \rightarrow \infty}(\tilde{f}(u) / u)=0$. Namely, the authors exploited the $C^{1}(\bar{\Omega})$ solution of

$$
\begin{gathered}
-\Delta_{p} z_{\alpha}-\alpha z_{\alpha}^{p-1}=-1 \quad \text { in } \Omega, \\
z_{\alpha}=0 \text { on } \partial \Omega,
\end{gathered}
$$


which is positive in $\Omega$ by the anti-maximum principle for $\alpha \in\left(\lambda_{1}, \lambda_{1}+\nu\right)$ for some $\nu>0$ where $\lambda_{1}$ is the first eigenvalue of the $-\Delta_{p}$ with Dirichlet boundary conditions. However this requires a further restriction on $\tilde{f}$ namely: there exists $m>0$ such that $\tilde{f}(v)>$ $v^{p-1}-m^{p-1} \alpha^{p-2}+(c / \alpha), \forall v \in\left[0, m \alpha\left\|z_{\alpha}\right\|_{\infty}\right]$. Moreover they obtain a positive a solution for $\lambda$ near the first eigenvalue $\lambda_{1}$. In proving Theorem 1.1 we avoid the use of the antimaximum principle in creating a crucial subsolution. Thus we avoid this above restriction on $f$ for small $u$ which seems unnatural when we look for positive solutions for large $\lambda$. In Theorem 1.1 we establish a subsolution by analyzing an appropriate power of the first eigenfunction of the $-\Delta_{p}$ with Dirichlet boundary conditions.

Also recently in [13] the Logistic equation with constant yield harvesting was studied via an anti-maximum principle in the case when $a(x)$ is a positive constant equal to $A_{0}$ $\left(>\lambda_{1}\right)$ throughout $\bar{\Omega}$. But in the case of Theorem 1.4, since we allow $a(x)$ to be negative near the boundary, the idea in [13] fails. Again we use an appropriate power of the eigenfunction to create the crucial subsolution needed to establish Theorem 1.4. We will prove Theorem 1.1 in Section 2 and Theorem 1.4 in Section 3.

\section{Proof of Theorem 1.1}

Here note that $g(x, u, c)=\lambda f(u)-c$ where $f$ satisfies (A1). Let $\lambda_{1}, \phi_{1}, \delta, m, \sigma$, and $\Omega_{\delta}$ be as described in Section 1.

We now construct our positive subsolution. Let $\psi:=((p-1) / p) r \phi_{1}^{p /(p-1)}$. (Note that $\|\psi\|_{\infty}<r$.) Then $\nabla \psi=r \phi_{1}^{1 /(p-1)} \nabla \phi_{1}$ and $\psi$ will be a subsolution if

$$
\int_{\Omega}|\nabla \psi|^{p-2} \nabla \psi \cdot \nabla w d x \leq \int_{\Omega}[\lambda f(\psi)-c] w d x, \quad \forall w \in W
$$

But

$$
\begin{aligned}
\int_{\Omega}|\nabla \psi|^{p-2} \nabla \psi \cdot \nabla w d x & =r^{p-1} \int_{\Omega}\left|\nabla \phi_{1}\right|^{p-2} \phi_{1} \nabla \phi_{1} \cdot \nabla w d x \\
& =r^{p-1}\left[\int_{\Omega}\left|\nabla \phi_{1}\right|^{p-2} \nabla \phi_{1} \cdot \nabla\left(\phi_{1} w\right) d x-\int_{\Omega}\left|\nabla \phi_{1}\right|^{p} w d x\right] \\
& =r^{p-1} \int_{\Omega}\left[\lambda_{1} \phi_{1}^{p}-\left|\nabla \phi_{1}\right|^{p}\right] w d x .
\end{aligned}
$$

Now $r^{p-1}\left[\lambda_{1} \phi_{1}^{p}-\left|\nabla \phi_{1}\right|^{p}\right] \leq-m r^{p-1}$ in $\bar{\Omega}_{\delta}$. Hence if $c \leq c_{0}=m r^{p-1}$ then $r^{p-1}\left[\lambda_{1} \phi_{1}^{p}-\right.$ $\left.\left|\nabla \phi_{1}\right|^{p}\right] \leq[\lambda f(\psi)-c]$ in $\bar{\Omega}_{\delta}$, since $f(\psi) \geq 0$.

Next in $\Omega-\bar{\Omega}_{\delta}, r^{p-1}\left[\lambda_{1} \phi_{1}^{p}-\left|\nabla \phi_{1}\right|^{p}\right] \leq \lambda_{1} r^{p-1}$ while

$$
\lambda f(\psi)-c \geq \lambda \alpha-c
$$

where $\alpha=\inf \left\{f(s) \mid((p-1) / p) r \sigma^{p /(p-1)} \leq s \leq((p-1) / p) r\right\}$. Hence if $\lambda \geq \lambda^{*}=\left(\lambda_{1} r^{p-1}+\right.$ c)/ $\alpha$ then in $\Omega-\bar{\Omega}_{\delta}$,

$$
r^{p-1}\left[\lambda_{1} \phi_{1}^{p}-\left|\nabla \phi_{1}\right|^{p}\right] \leq \lambda f(\psi)-c
$$

Hence if $c \leq c_{0}$ and $\lambda \geq \lambda^{*}$ then (2.1) is satisfied and $\psi$ is a subsolution. 
We next construct a super-solution $\phi$ such that $\phi \geq \psi$. Let $\phi:=M \phi_{0}$ where $\phi_{0} \in C^{1}(\Omega)$ is the solution of

$$
\begin{gathered}
-\Delta_{p} \phi_{0}=1 \quad \text { in } \Omega, \\
\phi_{0}=0 \quad \text { on } \partial \Omega .
\end{gathered}
$$

Now $\phi$ will be a super-solution if

$$
\int_{\Omega}|\nabla \phi|^{p-2} \nabla \phi \cdot \nabla w d x \geq \int_{\Omega}[\lambda f(\phi)-c] w d x, \quad \forall w \in W .
$$

But $\int_{\Omega}|\nabla \phi|^{p-2} \nabla \phi \cdot \nabla w d x=M^{p-1} \int_{\Omega} w d x \geq \int_{\Omega}[\lambda f(\phi)-c] w d x$, provided $M^{p-1} \geq \lambda$ $\sup _{[0, r]} f(s):=M(\lambda)$ (say). That is, if $M \geq(M(\lambda))^{1 /(p-1)}$ then (2.6) is satisfied and $\phi$ is a super-solution. Since $\phi_{0}>0$ in $\Omega$ and $\partial \phi_{0} / \partial n<0$ on $\partial \Omega$, we can choose $M$ large enough so that $\phi \geq \psi$ is also satisfied. Hence Theorem 1.1 is proven.

Remark 2.1. We have, in the proof of Theorem 1.1, an explicit expression for both $c_{0}(\Omega, r)$ and $\lambda^{*}(\Omega, r, c)$.

\section{Proof of Theorem 1.4}

Here note that $g(x, u, c)=a(x) u^{p-1}-u^{\gamma-1}-\operatorname{ch}(x)$. Let $\lambda_{1}, \phi_{1}, m, \sigma, \delta, a_{0}, \mu$, and $\Omega_{\delta}$ be as described in Section 1.

Let $\psi=\varepsilon \phi_{1}^{p /(p-1)}$ where $\varepsilon$ will be chosen small enough later. (Note that $\|\psi\|_{\infty} \leq \varepsilon$.) Then $\psi$ will be a subsolution if

$$
\int_{\Omega}|\nabla \psi|^{p-2} \nabla \psi \cdot \nabla w d x \leq \int_{\Omega}\left[a(x) \psi^{p-1}-\psi^{\gamma-1}-\operatorname{ch}(x)\right] w d x, \quad \forall w \in W .
$$

Using a calculation similar to the one in the proof of Theorem 1.1, we have

$$
\int_{\Omega}|\nabla \psi|^{p-2} \nabla \psi \cdot \nabla w d x=\varepsilon^{p-1}\left(\frac{p}{p-1}\right)^{p-1} \int_{\Omega}\left[\lambda_{1} \phi_{1}^{p}-\left|\nabla \phi_{1}\right|^{p}\right] w d x .
$$

Hence inequality (3.1) will be satisfied if both

$$
\begin{gathered}
\varepsilon^{p-1}\left(\frac{p}{p-1}\right)^{p-1}(-m) \leq-\mu \varepsilon^{p-1}-\varepsilon^{\gamma-1}-c \quad\left(\text { considering } \bar{\Omega}_{\delta}\right), \\
\varepsilon^{p-1}\left(\frac{p}{p-1}\right)^{p-1} \lambda_{1} \phi_{1}^{p} \leq a_{0} \varepsilon^{p-1} \phi_{1}^{p}-\varepsilon^{\gamma-1}-c \quad\left(\text { considering } \Omega \backslash \bar{\Omega}_{\delta}\right)
\end{gathered}
$$

are satisfied. Note that since $\mu<m(p /(p-1))^{p-1}$ inequality (3.3) will be satisfied if

$$
\begin{aligned}
\varepsilon<\alpha_{1} & =\left\{m\left(\frac{p}{p-1}\right)^{p-1}-\mu\right\}^{1 /(\gamma-p)}, \\
c \leq \widetilde{c}_{1}(\varepsilon) & =\varepsilon^{p-1}\left\{m\left(\frac{p}{p-1}\right)^{p-1}-\mu-\varepsilon^{\gamma-p}\right\} .
\end{aligned}
$$


Note that $\tilde{c}_{1}(\varepsilon)>0$. Similarly, since $a_{0}>(p /(p-1))^{p-1} \lambda_{1}$, inequality (3.4) will be satisfied if

$$
\begin{gathered}
\varepsilon \leq \alpha_{2}\left[\left\{a_{0}-\left(\frac{p}{p-1}\right)^{p-1} \lambda_{1}\right\} \sigma^{p}\right]^{1 /(\gamma-p)}, \\
c \leq \tilde{c}_{2}(\varepsilon)=\varepsilon^{p-1}\left[\left\{a_{0}-\left(\frac{p}{p-1}\right)^{p-1} \lambda_{1}\right\} \sigma^{p}-\varepsilon^{\gamma-p}\right] .
\end{gathered}
$$

Note that $\tilde{c}_{2}(\varepsilon)>0$. Choose $\alpha=\min \left\{\alpha_{1}, \alpha_{2}\right\}$ and $\varepsilon=\alpha / 2$. Then simplifying, both $\tilde{c}_{1}(\varepsilon)$ and $\tilde{c}_{2}(\varepsilon)$ are greater than $(\alpha / 2)^{\gamma-1}\left[2^{\gamma-p}-1\right]$. Hence if $c \leq(\alpha / 2)^{\gamma-1}\left[2^{\gamma-p}-1\right]=c_{1}\left(\Omega, a_{0}, \mu\right)$ then $\psi$ is a subsolution.

We next construct a super-solution $\phi$ such that $\phi \geq \psi$. Let $\phi:=M \phi_{0}$ where $\phi_{0} \in C^{1}(\bar{\Omega})$ is the solution of (2.5). Now $\phi$ will be a super-solution if

$$
\int_{\Omega}|\nabla \phi|^{p-2} \nabla \phi \cdot \nabla w d x \geq \int_{\Omega}\left[a(x) \phi^{p-1}-\phi^{\gamma-1}-\operatorname{ch}(x)\right] w d x, \quad \forall w \in W .
$$

But $\int_{\Omega}|\nabla \phi|^{p-2} \nabla \phi \cdot \nabla w d x=M^{p-1} \int_{\Omega} w d x \geq \int_{\Omega}\left[a(x) \phi^{p-1}-\phi^{\gamma-1}-\operatorname{ch}(x)\right] w d x$, provided $M^{p-1} \geq \sup _{[0, k]}\left[\|a\|_{\infty} s^{p-1}-s^{\gamma-1}\right]:=M_{1}$ (say) where $k=\|a\|_{\infty}^{1 /(\gamma-p)}$. That is, if $M \geq M_{1}^{1 /(p-1)}$ then (3.7) is satisfied and $\phi$ is a super-solution. Since $\phi_{0}>0$ in $\Omega$ and $\partial \phi_{0} / \partial n<0$ on $\partial \Omega$, we can choose $M$ large enough so that $\phi \geq \psi$ is also satisfied. Hence Theorem 1.4 is proven.

Remark 3.1. We have, in the proof of Theorem 1.4, an explicit expression for $c_{1}\left(\Omega, a_{0}, \mu\right)$.

\section{References}

[1] H. Berestycki, L. A. Caffarelli, and L. Nirenberg, Further qualitative properties for elliptic equations in unbounded domains, Annali della Scuola Normale Superiore di Pisa. Classe di Scienze. Serie IV 25 (1997), no. 1-2, 69-94, dedicated to E. De Giorgi.

[2] K. J. Brown and R. Shivaji, Simple proofs of some results in perturbed bifurcation theory, Proceedings of the Royal Society of Edinburgh. Section A. Mathematics 93 (1982), no. 1-2, 71-82.

[3] A. Castro, C. Maya, and R. Shivaji, Nonlinear eigenvalue problems with semipositone structure, Proceedings of the Conference on Nonlinear Differential Equations (Coral Gables, Fla, 1999), Electron. J. Differ. Equ. Conf., vol. 5, Southwest Texas State University, Texas, 2000, pp. 33-49.

[4] M. Chhetri, S. Oruganti, and R. Shivaji, Positive solutions for classes of p-Laplacian equations, Differential and Integral Equations 16 (2003), no. 6, 757-768.

[5] Ph. Clément and L. A. Peletier, An anti-maximum principle for second-order elliptic operators, Journal of Differential Equations 34 (1979), no. 2, 218-229.

[6] Ph. Clément and G. Sweers, Existence and multiplicity results for a semilinear elliptic eigenvalue problem, Annali della Scuola Normale Superiore di Pisa. Classe di Scienze. Serie IV 14 (1987), no. $1,97-121$.

[7] P. Drábek and J. Hernández, Existence and uniqueness of positive solutions for some quasilinear elliptic problems, Nonlinear Analysis 44 (2001), no. 2, 189-204.

[8] P. Drábek, P. Krejčí, and P. Takáč, Nonlinear Differential Equations, Chapman \& Hall/CRC Research Notes in Mathematics, vol. 404, Chapman \& Hall/CRC, Florida, 1999.

[9] J. Fleckinger-Pellé and P. Takáč, Uniqueness of positive solutions for nonlinear cooperative systems with the p-Laplacian, Indiana University Mathematics Journal 43 (1994), no. 4, 1227-1253. 
[10] D. D. Hai, On a class of sublinear quasilinear elliptic problems, Proceedings of the American Mathematical Society 131 (2003), no. 8, 2409-2414.

[11] D. D. Hai and R. Shivaji, Existence and uniqueness for a class of quasilinear elliptic boundary value problems, Journal of Differential Equations 193 (2003), no. 2, 500-510.

[12] S. Oruganti, J. Shi, and R. Shivaji, Diffusive logistic equation with constant yield harvesting. I. Steady states, Transactions of the American Mathematical Society 354 (2002), no. 9, 3601-3619.

[13] _ Logistic equation wtih the p-Laplacian and constant yield harvesting, Abstract and Applied Analysis 2004 (2004), no. 9, 723-727.

Shobha Oruganti: Department of Mathematics, School of Science, The Behrend College,

Penn State Erie, Erie, PA 16563, USA

E-mail address: sxo12@psu.edu

R. Shivaji: Department of Mathematics and Statistics, Mississippi State University, Mississippi State, MS 39762, USA

E-mail address: shivaji@ra.msstate.edu 